\title{
Lyophilized mRNA-lipid nanoparticle vaccines with long-term stability and
}

\section{high antigenicity against SARS-CoV-2}

Liangxia $\mathrm{Ai}^{1,{ }^{+}}$, Yafei $\mathrm{Li}^{1,{ }^{+}}$, Li Zhou ${ }^{2,{ }^{+}}$, Hao Zhang ${ }^{1}$, Wenrong $\mathrm{Yao}^{3}$, Jinyu Han ${ }^{1}$, Junmiao $\mathrm{Wu}^{1}$, Ruiyue Wang ${ }^{1}$, Weijie Wang ${ }^{1}$, Pan $\mathrm{Xu}^{1}$, Zhouwang $\mathrm{Li}^{1}$, Chengliang $\mathrm{Wei}^{1}$, Haobo Chen ${ }^{1}$, Jianqun Liang$^{1}$, Ming Guo ${ }^{4}$, Zhixiang Huang ${ }^{2}$, Xin Wang ${ }^{4}$, Zhen Zhang ${ }^{4}$, Wenjie Xiang ${ }^{4}$, Bin Lv ${ }^{1}$, Peiqi Peng ${ }^{1}$, Shangfeng Zhang ${ }^{1}$, Xuhao $\mathrm{Ji}^{1}$, Zhangyi $\mathrm{Li}^{1}{ }^{1}$, Huiyi Luo ${ }^{1}$, Jianping Chen ${ }^{3,5,}{ }^{*}$, Ke Lan $^{2,4}{ }^{,}$, Yong $\mathrm{Hu}^{1,5, *}$

${ }^{1}$ Shenzhen Rhegen Biotechnology Co. Ltd, Shenzhen 518057, China

2 Animal Biosafety Level 3 Laboratory/Institute for Vaccine Research, Wuhan University, Wuhan 430071, China

3 Jiangsu Rec-biotechnology Co. Ltd, Taizhou 225300, China

${ }^{4}$ State Key Laboratory of Virology, College of Life Sciences, Wuhan University, Wuhan 430071, China

${ }^{5}$ Wuhan Recogen Biotechnology Co. Ltd, Wuhan 430056, China

† These authors contributed equally to this work.

*Corresponding author: Ke Lan (Email: klan@whu.edu.cn)

Yong Hu (Email: yong.hu@rhegen.com)

\section{Abstract}

Advanced mRNA vaccines play vital roles against SARS-CoV-2. However, due to the poor stability, most current mRNA delivery platforms need to be stored at $-20^{\circ} \mathrm{C}$ or $-70^{\circ} \mathrm{C}$. Here we present lyophilized thermostable mRNA loaded lipid nanoparticles, which could be stored at room temperature with long-term stability. We demonstrate the applicability of lyophilization techniques to different mRNA sequences and lipid components. Three lyophilized vaccines targeting wild-type, Delta and Omicron SARS-CoV-2 variant were prepared and demonstrated to be able induce high-level of IgG titer and neutralization response. In the Delta challenge in vivo experiment, the lyophilized mRNA vaccine successfully protected the mice from infection and clear the virus. This lyophilization platform could significantly improve the accessibility of mRNA vaccine or therapeutics, particularly in remote regions. 
Keywords:

SARS-CoV-2, mRNA vaccine, Lyophilization, Lipid nanoparticles.

\section{Introduction}

After years of research and development, mRNA delivery systems made their breakthrough and became the frontrunners that prevent coronavirus disease 2019 (COVID-19). Compared with inactivated vaccines and recombinant protein vaccine, mRNA vaccines could be easily and quickly updated to target different variants, and with comparable first-class protective efficacy as well [1]. The sequence-independent manufacturing process saved a lot of time and cost to develop a new vaccine, especially in the pandemic situation, just as the situation fighting against the new variants of severe acute respiratory syndrome coronavirus 2 (SARSCoV-2) $[2,3]$.

Current mRNA therapeutics heavily depend on lipid or lipid-like delivery systems to improve their in vivo transfection efficacy [4]. Several lipid components form the nanoparticles carrying the mRNA to targeted organelles. Although mRNA therapeutics show such superiority, challenges about stability still impede their accessibility. Cryogenic preservation and transportation are needed for two current licensed mRNA vaccines, BNT162 b2 $\left(-80^{\circ} \mathrm{C} \sim-60^{\circ} \mathrm{C}\right)$ and mRNA-1273 $\left(-20^{\circ} \mathrm{C}\right)$ [5]. The demanding requirements come from the complex interactions among multiple lipid components and the instability of mRNA, which is sensitive to oxygen, moisture, enzymes, $\mathrm{pH}$ and even more [6]. As the exact mechanisms have not been solved, how to improve the stability of mRNA therapeutics is still challenging [7].

Lyophilization is a process that removes water by sublimation under vacuum at a low temperature [8]. It's a relatively mild drying method and could improve the stability of vulnerable macrobiomolecules or colloidal nanoparticles [9]. The lyophilized mRNA could be stored at $4{ }^{\circ} \mathrm{C}$ or at room temperature for long time $[10,11]$. However, drying of mRNA-lipid nanoparticles (mRNA-LNP) is more sophisticated, as the freezing and the dehydration process would induce mechanical force and deform the vehicle structure, leading to vehicle aggregation, mRNA breakage or leakage [12]. Moreover, some research showed that even the mRNA-LNP remained their integrity and encapsulation efficiency, the in vivo transfection efficacy was greatly reduced after lyophilization due to some unknown reasons [13].

Here we present an optimized lyophilization technique, which could effectively sustain the physiochemical properties and bioactivity of mRNA-LNP and achieve long-term storage at $2{ }^{\circ} \mathrm{C}$ 
$\sim 8{ }^{\circ} \mathrm{C}$. The improved thermostability was verified with LNPs containing different mRNA molecules, demonstrating its wide applicability. Furthermore, we utilized this technique to prepare the first lyophilized, thermostable mRNA-LNP vaccines with encoding the antigen of wild-type (WA1, Lyo-mRNA-WT), Delta (Lyo-mRNA-Delta) or Omicron COVID-19 variant (LyomRNA-Omicron) and confirmed their high-leveled antibody responses and prevention ability. Noticeably, we optimized the mRNA sequences in theses lyophilized mRNA vaccines. The lyophilized vaccine could both induce strong immune response and produce high levels of neutralizing antibody titer. The mRNA-Delta and mRNA-Omicron vaccine also showed crossprotection ability against other variants. In the challenge study of mRNA-Dela vaccine against Delta strain, the vaccine also demonstrated to fully protect the mice from infection and clear the virus.

\section{Materials and methods}

\subsection{Materials}

Lipids were purchased from Xiamen Sinopeg Biotech Co., Ltd. Cholesterol was purchased from AVT (Shanghai) Pharmaceutical Tech Co., Ltd. Ethanol was obtained from Aladdin. Citric acid and trisodium citrate were obtained from Sigma-aldrich. SARS-COV-2 WA1, Delta and Omicron pseudovirus using recombinant replication-deficient vesicular stomatitis virus (VSV) vector that encodes Luciferase instead of VSV-G (VSVDG-Luc) were obtained from Vazyme.

\subsection{RNA synthesis}

SARS-COV-2 NTD-RBD region were used as antigens in this work. The respective mRNAs were produced in vitro by standard T7 RNA polymerase-mediate transcription reaction and added with Cap1, then purified though Fast Protein Liquid Chromatography. Luciferase mRNA were

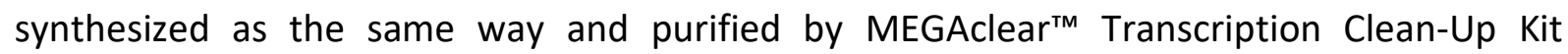
(Invitrogen).

\subsection{Lipid nanoparticle (LNP) preparation and characterization}

The lipid nanoparticles (LNPs) were prepared by mixing an aqueous phase containing the mRNA with an ethanol phase containing the lipid mixtures using a T-junction mixing device as reported previously [14]. In brief, the mRNA was dissolved in a $100 \mathrm{mM}$ citric buffer (pH 4.0). The lipid mixture was dissolved in anhydrous ethanol with the molar ratio of ionizable lipid:1, 
2-distearoyl-sn-glycero-3-phosphocholine (DSPC): cholesterol: PEG-lipid=46.3:9.4:42.7:1.6. The N:P ratio was kept as 6:1. And the ethanol and aqueous phases were mixed at a volume ratio of 3:1 in the T-junction device. After mixing, LNPs were dialyzed against a buffer at $\mathrm{pH}$ 7.4 for 6 hours. After that, LNPs were sterilized via a $0.22 \mu \mathrm{m}$ filter and stored at $4{ }^{\circ} \mathrm{C}$ for further use. The average diameter, polydispersity (PDI) and zeta potential were measured with a NS-90Z device (Malvern Panalytical). The cryo-TEM images were obtained from Southern University of Science and Technology. The concentration of leaked mRNA ( $C_{\text {leak }}$ ) was determined with a fluorescence detection kit, following the manufacture's protocols. LNP was also lysed with $0.4 \%$ Triton $\mathrm{X}-100$ to determine the concentration of total mRNA $\left(C_{\text {total }}\right)$. The encapsulation efficiency was calculated as the following equation:

$$
\text { Encapsulation efficiency }=\frac{C_{\text {total }}-C_{\text {leak }}}{C_{\text {total }}} \times 100 \%
$$

\subsection{Lyophilization}

LNP solution was added with cryoprotectant and filled in a penicillin bottle, then the mixture was lyophilized with a freezer dryer (Pilot-2H, Boyikang) using a designed procedure. The resulting powder was collected, characterized and stored at $4{ }^{\circ} \mathrm{C}$ for further use. To measure the stability, lyophilized LNPs were incubated at either $4{ }^{\circ} \mathrm{C}, 25^{\circ} \mathrm{C}$, or $40^{\circ} \mathrm{C}$ for different time. The mRNA integrity was measured with a gel retardation assay [15] and microfluidic capillary electrophoresis (Agilent Fragment Analyser) [16] , as previously reported.

\subsection{Cell culture}

HEK 293T/17 and ACE2-expressing 293T cells were cultured in Dulbecco's Modified Eagle's Medium (DMEM) supplemented with $2 \mathrm{mM}$ L-glutamine, 10\% FBS (sigma-aldrich) and 1\% penicillin/streptomycin (BI) at $37^{\circ} \mathrm{C}$ and $5 \% \mathrm{CO}_{2}$.

\subsection{Animals}

Female BALB/C mice age of 6-8 weeks and heterozygous B6/JGpt-H11 em1Cin(K18-ACE2)/Gpt mice (K18-hACE2 KI mice) age of 5-7 weeks and body weight of 18-20 g were used. The mice were housed under a $12 \mathrm{~h}$ dark-light cycle at a constant temperature. Animal experiments were executed by certified staff in Center for Animal Experiments of Wuhan University, approved by the Institutional Animal Care and Use Committee (AUP \#WP2021-0607). And the protocols 
and procedures of infectious SARS-CoV-2 virus under Animal Biosafety Level-III Laboratory facility were approved by the Institutional Biosafety Committee (IBC, Protocol \#S01322010B). All samples' inactivation was performed according to IBC approved standard procedures for removal of specimens from high containment.

\subsection{Luciferase transfection in vivo}

Luciferase mRNA loaded LNPs (mRNA-Luc LNPs) and its lyophilized product, Lyo-mRNA-Luc LNPs were examined with their in vivo transfection efficiency. The encapsulated mRNA concentration was adjusted to $100 \mu \mathrm{g} / \mathrm{mL}$ and freeze-dried. Lyo-mRNA-Luc LNPs were reconstituted using the same volume of nuclease-free water with before drying. Mice were injected with $100 \mu \mathrm{L}$ LNP solution through tail vain injection. Post 24-hour injection, mice were narcotized with avertin solution $(0.2 \%, 350 \mu \mathrm{L})$. Then the luciferin solution $(1.4 \%, 200 \mu \mathrm{L})$ was administered intraperitoneally. And the mice were imaged with an IVIS Spectrum in vivo imaging system (FluoView400, Boluteng).

\subsection{Immunization}

Three kinds of LNPs with mRNA encoding the antigen of WA1 (Lyo-mRNA-WT), Delta (LyomRNA-Delta) or Omicron COVID-19 variant (Lyo-mRNA-Omicron) were prepared and used for mice immunization experiment. $0.5 \mathrm{~mL}$ LNP solutions containing $0.1 \mathrm{mg} / \mathrm{mL}$ encapsulated mRNA were freeze-dried with a penicillin bottle. Lyophilized LNPs were reconstituted using $0.5 \mathrm{~mL}$ nuclease-free water. Mice were received two doses of LNPs through intramuscular injections of $20 \mu \mathrm{L}, 50 \mu \mathrm{L}$, or $100 \mu \mathrm{L}$ reconstituted LNPs. The dilution water was also nucleasefree. The blood samples were collected and centrifuged at $1200 \mathrm{~g}$ and $4{ }^{\circ} \mathrm{C}$ for $10 \mathrm{~min}$. The supernatant serum was separated, aliquoted, and frozen at $-80^{\circ} \mathrm{C}$.

\subsection{Enzyme-linked Immunosorbent Assay (ELISA)}

96-Well ELISA Microplates (Greiner) were coated with $2 \mathrm{ng} / \mu \mathrm{L}$ RBD protein (NOVOPROTEIN) in coating buffer (Dakewe) at $4^{\circ} \mathrm{C}$ for $15 \mathrm{~h}$. After washing and blocking, gradiently diluted mouse serum were incubated in plate at $4^{\circ} \mathrm{C}$ for $2 \mathrm{~h}$. Following washes, the secondary antibody, goat Anti-Mouse IgG H\&L conjugated HRP (Abcam), was incubated in plate at room

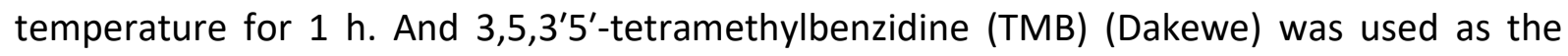
substrate to detect antibody responses. Data collection was performed using a MOLECULAR 
DEVICES microplate reader and SoftMax Pro software version 7.1.0. Endpoint titers were calculated as the dilution that absorbance exceeding $4 \mathrm{X}$ background (protein and secondary antibody).

\subsection{Pseudotyped virus neutralization assay}

Neutralizing antibody titers were tested according to the reported methods [17]. Briefly, serum samples were diluted and mixed with a certain amount $\left(1.3 \times 10^{4} \mathrm{TCID} / \mathrm{mL}\right)$ of pseudotyped virus and incubated at $37^{\circ} \mathrm{C}, 5 \% \mathrm{CO}_{2}$ for $30 \mathrm{~min}$. After that, the $293 \mathrm{~T}-\mathrm{ACE} 2$ cells are added and incubated for $24 \mathrm{~h}$. Then the Bio-Lite luciferase detection reagent (Vazyme) were mixed and incubated for $2 \mathrm{~min}$. The luminescence values (RLU) were detected with the microplate reader immediately, and the amount of pseudotyped virus entering cells was calculated by detecting the expression of luciferase, thus to obtain the neutralizing antibody content of the sample. Luminescence readout data are collected and the half maximal inhibitory concentration (IC50) are calculated for the tested samples.

\subsection{SARS-CoV-2 Delta virus neutralization assay}

SARS-CoV-2 Delta-neutralizing antibody was determined by in vitro inhibition of cytopathic effect (CPE), and was performed in the ABSL-3 lab at Center of Laboratory Animal Sciences, Wuhan University (Wuhan, China). Sera from K18-hACE2 KI mice were diluted in cell culture medium, and serial 3-fold dilutions starting from 1:100 to 1:218700. The diluted samples were mixed with a SARS-CoV-2 Delta virus suspension of 100 plaque-forming unit (PFU), followed by $1 \mathrm{~h}$ incubation. The virus-serum mixtures were added to Vero-E6 cells seeded in 24-well plate and cultured in a $5 \% \mathrm{CO}_{2}$ incubator at $37{ }^{\circ} \mathrm{C}$ for 3 days. The inhibitory capacity of each sample dilution was assessed for cytopathic effect (CPE), and the neutralization titer was calculated as the reciprocal of serum dilution required for $50 \%$ neutralization of viral infection.

\subsection{SARS-CoV-2 Delta challenge in K18-hACE2 KI transgenic mice}

The SARS-CoV-2 Delta challenge in K18-hACE2 KI transgenic mice to evaluate Lyo-mRNA-Delta vaccines of the study was performed at Animal Biosafety Level 3 Laboratory, Wuhan University. 36 female K18-hACE2 KI transgenic mice, 6 weeks old, were divided into 4 groups, namely the negative control group $(n=6)$, blank lipid nanoparticles (blank LNP) control group $(n=6)$, lowdose vaccine group $(n=12)$ ( $5 \mu \mathrm{g} /$ dose) and high-dose vaccine group ( $n=12)(10 \mu g / d o s e)$. The 
latter three groups of animals were intramuscularly immunized with $0.1 \mathrm{ml}$ blank LNP, $5 \mu \mathrm{g}$ Lyo-mRNA-Delta and $10 \mu \mathrm{g}$ Lyo-mRNA-Delta, respectively, at Day 0 (D0) and Day 14 (D14). At Day 28 (D28, 14 days after the second immunization), mice immunized with Lyo-mRNA-Delta or only blank LNP were challenged with $2.5 \times 10^{3}$ PFU SARS-CoV-2 Delta virus intranasally. The challenged mice were observed clinical symptoms and death, and weighed on day of infection and daily for up to 14 days post infection (dpi). At days 7 and $14 \mathrm{dpi}$, six mice were sacrificed under anesthesia from each vaccination, and $7 \mathrm{dpi}$ from blank LNP control group. The lung and brain tissues were collected to determine viral titer by qPCR.

\subsection{Statistical analysis}

Data were collected at least six independent experiments for in vivo experiment. All other experiments were performed at least three times independently unless specified. Statistical analysis was mainly processed using Prism software version 8.3 (GraphPad Software Inc., San Diego, CA) and analyzed by one-way or two-way analysis of variance (ANOVA) followed by Tukey's post-hoc test. Statistically significant differences were considered when $p<0.05$.

\section{Results and discussion}

\subsection{LNP preparation and lyophilization}

Four kinds of LNPs used in this paper were prepared through a classic T-junction mixing process [14]. LNPs containing Luc mRNA (mRNA-Luc LNPs), WT mRNA (mRNA-WT LNPs), Delta mRNA (mRNA-Delta LNPs) or Omicron (mRNA-Omicron LNPs) all showed narrow size distribution and high encapsulation efficiency (EE), as listed in Table 1. From the cryo-TEM images (Figure 1A), we could observe the uniform, lamellar and vesicular structure of the LNPs. After lyophilization, the dried LNPs looked like a white fluffy cake (Figure S1, supporting information) and could be dissolved in water easily and quickly ( $<10$ seconds). The reconstituted solution was uniform and translucent, just as the freshly-prepared LNP solutions. The size, polydispersity index (PDI) and encapsulation efficiency of LNPs (Table 1) only changed slightly, indicating that the optimized lyophilization process didn't changed their basic physical properties. The mRNA integrity was also well-maintained ( $>90 \%$, Figure S2, supporting information), demonstrating the lyophilization process didn't cause damage to the mRNA structure. 
We then evaluated the thermostability of Luc LNPs by incubating then at $4{ }^{\circ} \mathrm{C}, 25^{\circ} \mathrm{C}$, or at 40 ${ }^{\circ} \mathrm{C}$ for different time (Figure 1B, 1C). The products didn't exhibit any change and sustained the size and encapsulation efficiency at $4{ }^{\circ} \mathrm{C}$ and $25^{\circ} \mathrm{C}$ after 18 days, indicating the high stability of the product. At $40{ }^{\circ} \mathrm{C}$, the size increased, but the encapsulation efficiency and mRNA integrity (Figure 1D) were maintained. It could be estimated that the lyophilized LNPs could be stored at a $2 \sim 8{ }^{\circ} \mathrm{C}$ refrigerator for a long time.

The in vivo transfection efficiency of lyophilized LNPs was first evaluated with Luciferase mRNA. As seen in Figure 1E, mice treated with lyophilized mRNA-Luc LNPs (Lyo-mRNA-Luc LNPs) showed comparable total fluorescent intensity $\left(6.91 \pm 0.22 \times 10^{7} \mathrm{p} / \mathrm{s}\right)$ with those treated with freshly-prepared Luc LNPs $\left(9.43 \pm 0.66 \times 10^{7} \mathrm{p} / \mathrm{s}\right)$. This result indicated the mRNA-Luc LNPs maintained its bioactivity well after lyophilization. Next, we investigated the immunogenicity of mRNA-WT LNPs after lyophilization, following a prime-boost regimen. Mice were immunized with mRNA-WT LNPs or Lyo-mRNA-WT LNPs at day 0 and day 7, through intramuscular injection, then the serum was collected at day 21 . As shown in Figure $2 \mathrm{~A}$, all vaccines induced high IgG titers after immunization. The bioactivity of the antibodies was then tested through a pseudotyped virus assay (Figure 2B). Neutralizing response was also observed at this time point, titers against WA1 for the groups treated with $5 \mu \mathrm{g}$ mRNA-WT LNPs (1991) and $5 \mu \mathrm{g}$ Lyo-mRNA-WT LNPs (1973) showed no significant difference, demonstrating the lyophilization process didn't affect their bioactivity.

Table 1. Main physical characteristics of LNPs before and after lyophilization

\begin{tabular}{c|c|c|c|c|c|c}
\hline \multirow{2}{*}{ Samples } & \multicolumn{3}{|c|}{ Fresh } & \multicolumn{3}{c}{ Lyophilized } \\
\cline { 2 - 7 } & Size $(\mathrm{nm})$ & PDI & EE (\%) & Size $(\mathrm{nm})$ & PDI & EE (\%) \\
\hline mRNA-Luc LNPs & 82.1 & 0.12 & 95.2 & 116.8 & 0.13 & 87.9 \\
\hline mRNA-WT LNPs & 92.4 & 0.14 & 92.1 & 117.7 & 0.16 & 87.0 \\
\hline mRNA-Delta LNPs & 85.1 & 0.12 & 93.8 & 106.3 & 0.14 & 95.6 \\
\hline mRNA-Omicron LNPs & 77.6 & 0.09 & 94.3 & 88.4 & 0.16 & 86.2 \\
\hline
\end{tabular}



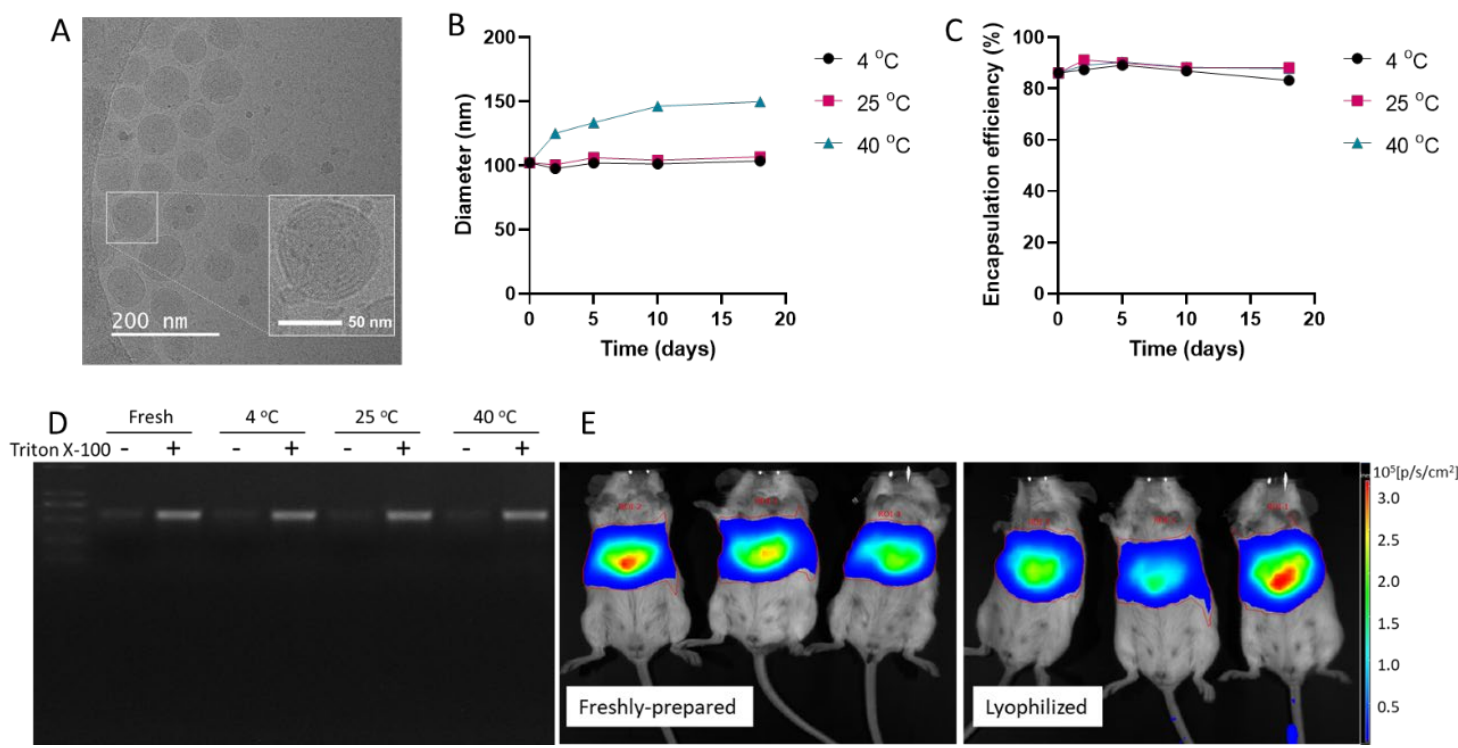

Figure 1. (A) Cryo-TEM image of mRNA-Luc LNPs. Change of (B) size and (C) encapsulation efficiency of Lyo-mRNA-Omicron LNPs at $4{ }^{\circ} \mathrm{C}$, $25{ }^{\circ} \mathrm{C}$, or $40{ }^{\circ} \mathrm{C}$ after being incubated for different time. (D) Stability of Lyo-mRNA-Omicron LNPs at $4^{\circ} \mathrm{C}, 25^{\circ} \mathrm{C}$ and $40^{\circ} \mathrm{C}$ after 10 days. Triton X-100 was used to release mRNA from LNPs. (E) Bioluminescent images of mice after treated with freshly-prepared or lyophilized Luc LNPs.
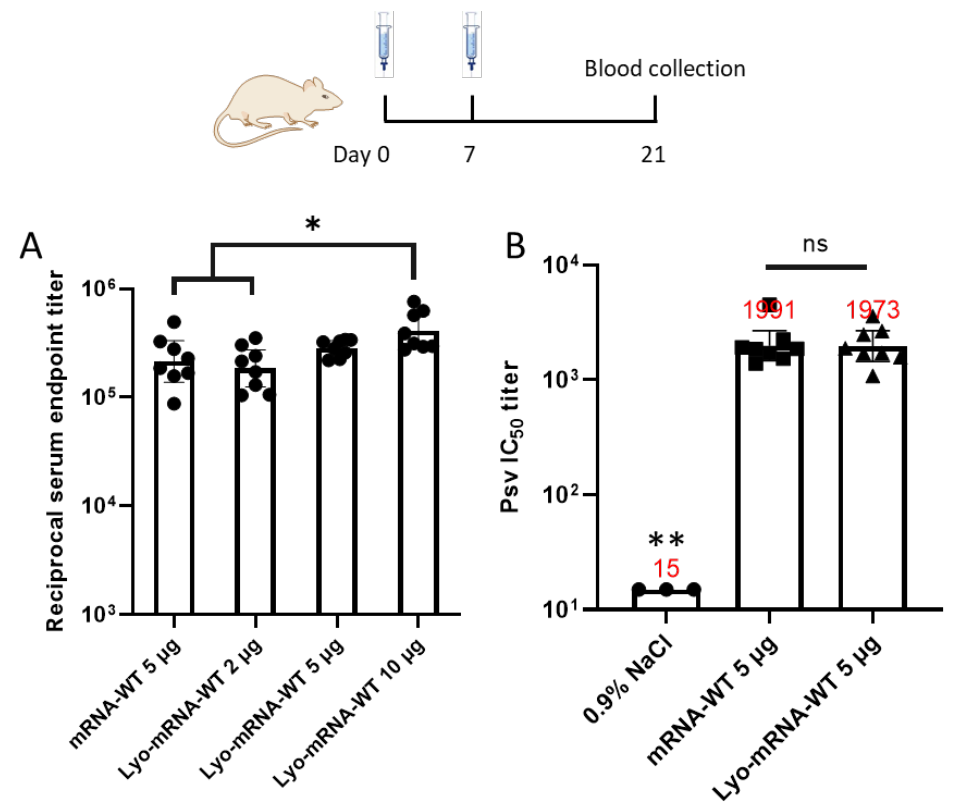

Figure 2. (A) Total IgG titer and (B) Pseudotyped $I C_{50}$ titer of mice post-immunized twice with freshly-prepared mRNA-WT LNPs or Lyo-mRNA-WT LNPs. ns: no significant difference; $* p<0.05, * * p<0.005$

\subsection{High neutralizing antibody induced by lyophilized SARS-COV-2 mRNA vaccine}


Next, a mRNA-Delta vaccine was prepared to further evaluate its efficacy against SARS-COV-2 Delta and other variants based on the lyophilized mRNA-LNP formulation (Lyo-mRNA-Delta). As show in Figure 3A, after immunizing intramuscularly twice with $5 \mu \mathrm{g}$ dosage at a 2-week interval, the sera obtained 14 days after the second vaccination showed a very strong DeltaRBD specific IgG antibody response in the six-week-old female BALB/cJ mice, with a geometric mean titer of $2.37 \times 10^{6}$. Potent neutralizing activity was also induced at this time point, titers against Delta were up to 9340 reciprocal ID50, while cross against Omicron, WA1 were 3278 and 30874, respectively, as showed in a vesicular stomatitis virus-based SARS-CoV-2 pseudovirus neutralization assay (Figure 3B). In contrast to the phenomenon of 20- to 130fold neutralization reduction for ancestral spike vaccines elicited sera [18-22], the titers of lyophilized mRNA-Delta vaccine against Omicron were still high, consistent with data from other studies $[23,24]$. Interestingly, the mRNA-Delta vaccine stimulated much higher neutralizing antibody titers to wild-type SARS-COV-2 pseudovirus than Delta itself.

We also developed a lyophilized mRNA vaccine against Omicron (Lyo-mRNA-Omicron) since it has a significant transmission advantage over Delta and become the dominant variant of concern in the current wave of COVID-19 [25]. To investigate its antigenicity, mice were twodose injected intramuscularly by an accelerated immunization process at day 0 and day 7 . Sera were collected one week after the second dose and pseudovirus titers were analyzed. Neutralizing antibody titers against Omicron increased to a high value as 4758 . Still, it could induce certain neutralization response against Delta though lower than Omicron.

In summary, our lyophilized mRNA vaccines were able to trigger robust antibody response against SARS-COV-2 virus. 

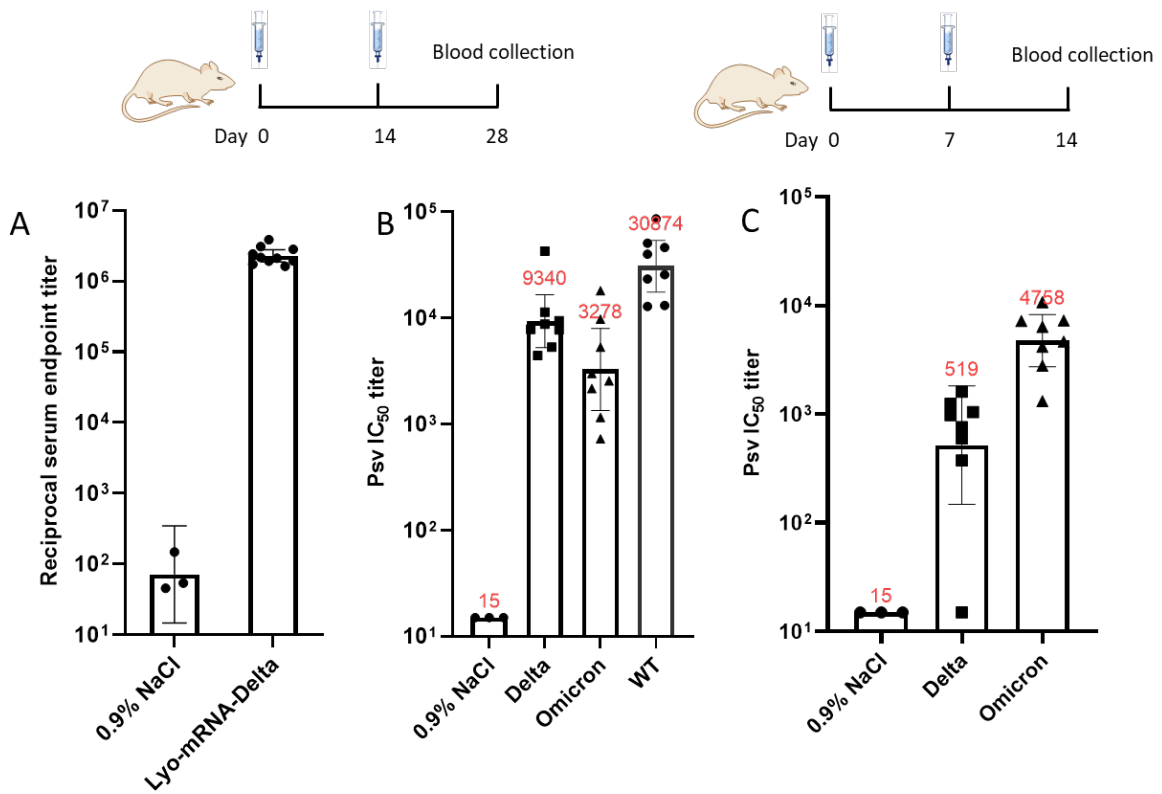

Figure 3. Lyophilized mRNA vaccine elicited robust binding and pseudovirus neutralizing antibody responses in BALB/c mice. Mice were immunized at day 0 and day 14 with LyomRNA-Delta, and sera were collected 14 days post-immunization. The (A) IgG titer and (B) neutralizing antibodies against Delta, Omicron or WT SARS-CoV-2 pseudovirus were analyzed. Other mice were immunized with an accelerated immune process at day 0 and day 7 with LyomRNA-Omicron, and sera were collected 7 days post-immunization. (C) The neutralizing antibodies against Delta and Omicron SARS-CoV-2 pseudovirus were analyzed.

\subsection{Protective efficacy of lyophilized mRNA-Delta vaccine in K18-hACE2 KI transgenic mice}

The immunogenicity and protective efficacy of Lyo-mRNA-Delta were evaluated in K18-hACE2 $\mathrm{KI}$ transgenic mice. The results showed that mice received $5 \mu \mathrm{g}$ and $10 \mu \mathrm{g}$ Lyo-mRNA-delta vaccine were sufficient to induce robust neutralizing activity before the challenge, the GMT of neutralizing antibodies against the SARS-CoV-2 Delta virus reached 4627 and 9044, respectively (Figure 4A). After challenge, K18-hACE2 KI mice received one-dose blank LNPs were highly susceptible to SARS-CoV-2 Delta infection. Beginning at $2 \mathrm{dpi}$, mice demonstrated clinical symptoms, including lethargy, ruffled fur, arched back, drowsiness. At 6 dpi, body weight reduction was seen in blank LNP control mice, and showed approximately $10 \%$ weight loss by $7 \mathrm{dpi}$, and one-thirds mice (two mice) died, and the remaining mice were moribund, humanely euthanized according to ethical principle. In contrast, mice received two-dose of reconstituted Lyo-mRNA-Delta showed no clinical abnormity, with no difference in body weight comparing to the negative control mice (Figure 4B, C). For these groups, half of the 
mice (six mice) were euthanized $7 \mathrm{dpi}$, and the other half (six mice) euthanized $14 \mathrm{dpi}$. After euthanasia, brain and right lung samples were collected from each animal for viral load quantification, and left lung were fixed for histopathological evaluation. The results showed that lyophilized mRNA-Delta vaccination protected the mice from SARS-CoV-2 Delta infection. The viral load in right lung tissue of Lyo-mRNA-Delta vaccinated animals remained the level of low limit of quantification $7 \mathrm{dpi}$. In contrast, a high level of viral load existed in the right lung tissue of animals in blank LNP control group, which up to approximately 1011 copies/g (Figure 4D). Importantly, the viral load in right lung tissue had almost undetectable in all LyomRNA-Delta vaccinated mice $14 \mathrm{dpi}$ (Below detection limit). The viral load in brain tissues in 5 $\mu \mathrm{g}$ animal groups displayed similar patterns as those in lung tissues. And $10 \mu \mathrm{g}$ Lyo-mRNADelta showed remarkable protective effect on viral dissemination and amplification (Figure $4 \mathrm{E})$.

Together, these data suggest that lyophilized mRNA-Delta vaccine is highly immunogenic and can fully protect the challenged mice from SARS-COV-2 infection.
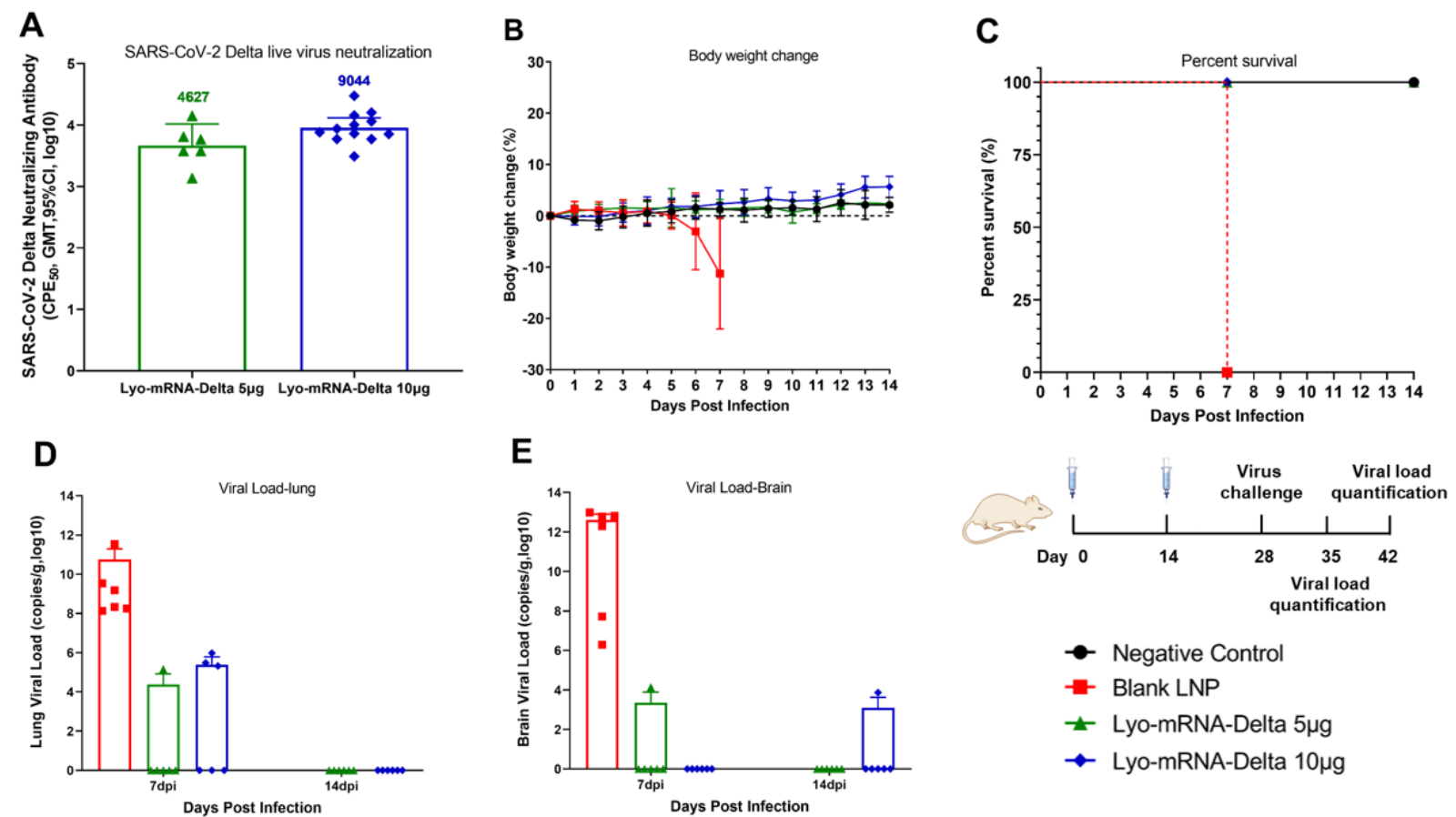

Figure 4. Protective efficacy of Lyo-mRNA delta vaccine in K18-hACE2 KI transgenic mice. Mice were intramuscularly inoculated with $5 \mu \mathrm{g}$ or $10 \mu \mathrm{g}$ Lyo-mRNA delta vaccine twice, separated by 14 days, and two weeks after the second dose, $2.5 \times 10^{3}$ PFU SARS-CoV-2 Delta was administrated to the animals intranasally. (A) Neutralizing antibody to SARS-CoV-2 delta virus induced by Lyo-mRNA-delta vaccine. (B) Changes in body weight after challenge. (C) Animal 
survival curve after challenge, all blank LNP animals died or were euthanized 7 days after challenge. (D) and (E) Viral load in lung and brain 7 and 14 days after challenge.

\section{Conclusion}

The mRNA vaccine has several advantages, such as rapid design of antigen, potential for quick, inexpensive and scalable manufacturing, and inducing strong humoral and cellular immunity, which make it a powerful weapon against infectious diseases, especially against those caused by virus that are easy to mutation. The advantage and efficacy of mRNA vaccines has also been proved by two approved and marketed mRNA vaccines against COVID-19, Comirnaty and Spikevax. While the storage and transport of the current approved mRNA vaccines require ultracold temperature, which limit its accessibility.

According to data in Our World in Data as of Feb. 6, 2022, 61.4\% of the world population has received at least one dose of a COVID-19 vaccine. 10.22 billion doses have been administered globally. However only $10 \%$ of people in low-income countries have received at least one-dose vaccine [26]. Therefore, it is particularly important to develop a mRNA vaccine that can be transported in conventional cold-chain and be stored at $2{ }^{\circ} \mathrm{C}^{\circ} \mathrm{C}$, or at room temperature stably for broad application.

In this study, we successfully achieved the lyophilization of mRNA-LNP vaccine. The particle size, encapsulation efficiency, poly dispersity index and zeta potential after reconstitution only changed slightly compared with freshly-prepared LNPs. And the bioactivity and immunogenicity were well-maintained, meeting the mRNA vaccine specification. The lyophilized mRNA-Delta and mRNA-Omicron vaccine could both produce high levels of neutralizing antibody titer. In the challenge study of Lyo-mRNA-Dela vaccine against Delta strain, it was confirmed that the vaccine could fully protect the mice from infection and clear the virus. Moreover, the lyophilized mRNA-LNPs showed long term storage stability at $4{ }^{\circ} \mathrm{C}$ and $25^{\circ} \mathrm{C}$, addressing the storage and transportation issue of current mRNA vaccines.

In conclusion, the lyophilized mRNA-LNP vaccine has the advantage of both high immunogenicity and accessibility, and very suitable for prevention of epidemic such as SARSCOV-2. We are actively promoting its clinical trial application.

\section{Supporting Information}

Supporting Information is available from the author. 
bioRxiv preprint doi: https://doi.org/10.1101/2022.02.10.479867; this version posted February 11, 2022. The copyright holder for this preprint (which was not certified by peer review) is the author/funder. All rights reserved. No reuse allowed without permission.

\section{References}

[1] J.T. Granados-Riveron, G. Aquino-Jarquin, Engineering of the current nucleoside-modified mRNA-LNP vaccines against SARS-CoV-2, Biomed Pharmacother 142 (2021) 111953.

[2] A.B. Vogel, I. Kanevsky, Y. Che, K.A. Swanson, A. Muik, M. Vormehr, L.M. Kranz, K.C. Walzer, S. Hein, A. Guler, J. Loschko, M.S. Maddur, A. Ota-Setlik, K. Tompkins, J. Cole, B.G. Lui, T. Ziegenhals, A. Plaschke, D. Eisel, S.C. Dany, S. Fesser, S. Erbar, F. Bates, D. Schneider, B. Jesionek, B. Sanger, A.K. Wallisch, Y. Feuchter, H. Junginger, S.A. Krumm, A.P. Heinen, P. Adams-Quack, J. Schlereth, S. Schille, C. Kroner, R. de la Caridad Guimil Garcia, T. Hiller, L. Fischer, R.S. Sellers, S. Choudhary, O. Gonzalez, F. Vascotto, M.R. Gutman, J.A. Fontenot, S. Hall-Ursone, K. Brasky, M.C. Griffor, S. Han, A.A.H. Su, J.A. Lees, N.L. Nedoma, E.H. Mashalidis, P.V. Sahasrabudhe, C.Y. Tan, D. Pavliakova, G. Singh, C. Fontes-Garfias, M. Pride, I.L. Scully, T. Ciolino, J. Obregon, M. Gazi, R. Carrion, Jr., K.J. Alfson, W.V. Kalina, D. Kaushal, P.Y. Shi, T. Klamp, C. Rosenbaum, A.N. Kuhn, O. Tureci, P.R. Dormitzer, K.U. Jansen, U. Sahin, BNT162b vaccines protect rhesus macaques from SARS-CoV-2, Nature 592(7853) (2021) 283-289.

[3] C. Garrido, A.D. Curtis, M. Dennis, S.H. Pathak, H. Gao, D. Montefiori, M. Tomai, C.B. Fox, P.A. Kozlowski, T. Scobey, SARS-CoV-2 vaccines elicit durable immune responses in infant rhesus macaques, Science immunology 6(60) (2021) eabj3684.

[4] Y. Liu, K.M. Castro Bravo, J. Liu, Targeted liposomal drug delivery: a nanoscience and biophysical perspective, Nanoscale Horiz 6(2) (2021) 78-94.

[5] D.J.A. Crommelin, T.J. Anchordoquy, D.B. Volkin, W. Jiskoot, E. Mastrobattista, Addressing the Cold Reality of mRNA Vaccine Stability, J Pharm Sci 110(3) (2021) 997-1001. [6] L. Schoenmaker, D. Witzigmann, J.A. Kulkarni, R. Verbeke, G. Kersten, W. Jiskoot, D.J.A. Crommelin, mRNA-lipid nanoparticle COVID-19 vaccines: Structure and stability, Int J Pharm 601 (2021) 120586.

[7] Y. Weng, C. Li, T. Yang, B. Hu, M. Zhang, S. Guo, H. Xiao, X.J. Liang, Y. Huang, The challenge and prospect of mRNA therapeutics landscape, Biotechnol Adv 40 (2020) 107534.

[8] W. Abdelwahed, G. Degobert, S. Stainmesse, H. Fessi, Freeze-drying of nanoparticles: formulation, process and storage considerations, Adv Drug Deliv Rev 58(15) (2006) 1688-713. [9] C. Chen, D. Han, C. Cai, X. Tang, An overview of liposome lyophilization and its future potential, Journal of Controlled Release 142(3) (2010) 299-311.

[10] A.L. Fabre, M. Colotte, A. Luis, S. Tuffet, J. Bonnet, An efficient method for long-term room temperature storage of RNA, Eur J Hum Genet 22(3) (2014) 379-85.

[11] K.L. Jones, D. Drane, E.J. Gowans, Long-term storage of DNA-free RNA for use in vaccine studies, Biotechniques 43(5) (2007) 675-81.

[12] Y. Wang, D.W. Grainger, Lyophilized liposome-based parenteral drug development: Reviewing complex product design strategies and current regulatory environments, Adv Drug Deliv Rev 151-152 (2019) 56-71.

[13] P. Zhao, X. Hou, J. Yan, S. Du, Y. Xue, W. Li, G. Xiang, Y. Dong, Long-term storage of lipid-like nanoparticles for mRNA delivery, Bioact Mater 5(2) (2020) 358-363.

[14] M.J.W. Evers, J.A. Kulkarni, R. van der Meel, P.R. Cullis, P. Vader, R.M. Schiffelers, State-of-the-Art Design and Rapid-Mixing Production Techniques of Lipid Nanoparticles for Nucleic Acid Delivery, Small Methods 2(9) (2018).

[15] Y. Qiu, R.C.H. Man, Q. Liao, K.L.K. Kung, M.Y.T. Chow, J.K.W. Lam, Effective mRNA pulmonary delivery by dry powder formulation of PEGylated synthetic KL4 peptide, J Control Release 314 (2019) 102-115.

[16] A.B. Vogel, I. Kanevsky, Y. Che, K.A. Swanson, A. Muik, M. Vormehr, L.M. Kranz, K.C. Walzer, S. Hein, A. Güler, A prefusion SARS-CoV-2 spike RNA vaccine is highly immunogenic and prevents lung infection in non-human primates, BioRxiv (2020). 
bioRxiv preprint doi: https://doi.org/10.1101/2022.02.10.479867; this version posted February 11, 2022. The copyright holder for this preprint (which was not certified by peer review) is the author/funder. All rights reserved. No reuse allowed without permission.

[17] J. Nie, Q. Li, J. Wu, C. Zhao, H. Hao, H. Liu, L. Zhang, L. Nie, H. Qin, M. Wang, Q. Lu, X. Li, Q. Sun, J. Liu, C. Fan, W. Huang, M. Xu, Y. Wang, Quantification of SARS-CoV-2 neutralizing antibody by a pseudotyped virus-based assay, Nat Protoc 15(11) (2020) 3699-3715. [18] M. Gagne, J.I. Moliva, K.E. Foulds, S.F. Andrew, B.J. Flynn, A.P. Werner, D.A. Wagner, I.T. Teng, B.C. Lin, C. Moore, N. Jean-Baptiste, R. Carroll, S.L. Foster, M. Patel, M. Ellis, V.V. Edara, N.V. Maldonado, M. Minai, L. McCormick, C.C. Honeycutt, B.M. Nagata, K.W. Bock, C.N.M. Dulan, J. Cordon, J.-P.M. Todd, E. McCarthy, L. Pessaint, A. Van Ry, B. Narvaez, D. Valentin, A. Cook, A. Dodson, K. Steingrebe, D.R. Flebbe, S.T. Nurmukhambetova, S. Godbole, A.R. Henry, F. Laboune, J. Roberts-Torres, C.G. Lorang, S. Amin, J. Trost, M. Naisan, M. Basappa, J. Willis, L. Wang, W. Shi, N.A. Doria-Rose, A.S. Olia, C. Liu, D.R. Harris, A. Carfi, J.R. Mascola, P.D. Kwong, D.K. Edwards, H. Andersen, M.G. Lewis, K.S. Corbett, M.C. Nason, A.B. McDermott, M.S. Suthar, I.N. Moore, M. Roederer, N.J. Sullivan, D.C. Douek, R.A. Seder, mRNA-1273 or mRNA-Omicron boost in vaccinated macaques elicits comparable $\mathrm{B}$ cell expansion, neutralizing antibodies and protection against Omicron, (2022).

[19] M. Hoffmann, N. Kruger, S. Schulz, A. Cossmann, C. Rocha, A. Kempf, I. Nehlmeier, L. Graichen, A.S. Moldenhauer, M.S. Winkler, M. Lier, A. Dopfer-Jablonka, H.M. Jack, G.M.N. Behrens, S. Pohlmann, The Omicron variant is highly resistant against antibody-mediated neutralization: Implications for control of the COVID-19 pandemic, Cell 185(3) (2022) 447$456 \mathrm{e} 11$.

[20] V.-V. Edara, K.E. Manning, M. Ellis, L. Lai, K.M. Moore, S.L. Foster, K. Floyd, M.E. Davis-Gardner, G. Mantus, L.E. Nyhoff, S. Bechnak, G. Alaaeddine, A. Naji, H. Samaha, M. Lee, L. Bristow, M. Gagne, J. Roberts-Torres, A.R. Henry, S. Godbole, A. Grakoui, M. Saxton, A. Piantadosi, J.J. Waggoner, D.C. Douek, N. Rouphael, J. Wrammert, M.S. Suthar, mRNA1273 and BNT162b2 mRNA vaccines have reduced neutralizing activity against the SARSCoV-2 omicron variant, Cell Reports Medicine (2022).

[21] F. Schmidt, F. Muecksch, Y. Weisblum, J. Da Silva, E. Bednarski, A. Cho, Z. Wang, C. Gaebler, M. Caskey, M.C. Nussenzweig, Plasma neutralization of the SARS-CoV-2 Omicron variant, New England Journal of Medicine (2021).

[22] A. Muik, B.G. Lui, A.-K. Wallisch, M. Bacher, J. Mühl, J. Reinholz, O. Ozhelvaci, N. Beckmann, R.d.l.C. Güimil Garcia, A. Poran, Neutralization of SARS-CoV-2 Omicron by BNT162b2 mRNA vaccine-elicited human sera, Science (2022) eabn7591.

[23] I.J. Lee, C.-P. Sun, P.-Y. Wu, Y.-H. Lan, I.H. Wang, W.-C. Liu, S.-C. Tseng, S.-I. Tsung, Y.-C. Chou, M. Kumari, Y.-W. Chang, H.-F. Chen, Y.-S. Lin, T.-Y. Chen, C.-W. Chiu, C.-H. Hsieh, C.-Y. Chuang, C.-C. Lin, C.-M. Cheng, H.-T. Lin, W.-Y. Chen, P.-C. Chiang, C.-C. Lee, J.C. Liao, H.-C. Wu, M.-H. Tao, Omicron-specific mRNA vaccine induced potent neutralizing antibody against Omicron but not other SARS-CoV-2 variants, (2022).

[24] L. Qu, Z. Yi, Y. Shen, L. Lin, F. Chen, Y. Xu, Z. Wu, H. Tang, X. Zhang, F. Tian, C. Wang, X. Xiao, X. Dong, L. Guo, S. Lu, C. Yang, C. Tang, Y. Yang, W. Yu, J. Wang, Y. Zhou, Q. Huang, A. Yisimayi, Y. Cao, Y. Wang, Z. Zhou, X. Peng, J. Wang, X.S. Xie, W. Wei, Circular RNA Vaccines against SARS-CoV-2 and Emerging Variants, (2022).

[25] R. Viana, S. Moyo, D.G. Amoako, H. Tegally, C. Scheepers, C.L. Althaus, U.J. Anyaneji, P.A. Bester, M.F. Boni, M. Chand, Rapid epidemic expansion of the SARS-CoV-2 Omicron variant in southern Africa, Nature (2022) 1-10.

[26] https://ourworldindata.org/covid-vaccinations. 


\section{Supporting Information}

\section{Lyophilized mRNA-lipid nanoparticles vaccine with long-term stability and high antigenicity against SARS-CoV-2}

Liangxia $\mathrm{Ai}^{1,{ }^{+}}$, Yafei $\mathrm{Li}^{1,{ }^{+}}$, Li Zhou ${ }^{2,+}$, Hao Zhang ${ }^{1}$, Wenrong $\mathrm{Yao}^{3}$, Jinyu Han ${ }^{1}$, Junmiao $\mathrm{Wu}^{1}$, Ruiyue Wang ${ }^{1}$, Weijie Wang ${ }^{1}$, Pan $\mathrm{Xu}^{1}$, Zhouwang $\mathrm{Li}^{1}$, Chengliang $\mathrm{Wei}^{1}$, Haobo Chen ${ }^{1}$, Jianqun Liang$^{1}$, Ming Guo ${ }^{4}$, Zhixiang Huang ${ }^{2}$, Xin Wang ${ }^{4}$, Zhen Zhang ${ }^{4}$, Wenjie Xiang ${ }^{4}$, Bin Lv ${ }^{1}$, Peiqi Peng ${ }^{1}$, Shangfeng Zhang ${ }^{1}$, Xuhao $\mathrm{Ji}^{1}$, Zhangyi $\mathrm{Li}^{1}$, Huiyi Luo ${ }^{1}$, Jianping Chen ${ }^{3,5,}{ }^{*}, \mathrm{Ke} \mathrm{Lan}^{2,4,}{ }^{*}$, Yong $\mathrm{Hu}^{1,5, *}$

${ }^{1}$ Shenzhen Rhegen Biotechnology Co. Ltd, Shenzhen 518057, China

2 Animal Biosafety Level 3 Laboratory/Institute for Vaccine Research, Wuhan University, Wuhan 430071, China

3 Jiangsu Rec-biotechnology Co. Ltd, Taizhou 225300, China

${ }^{4}$ State Key Laboratory of Virology, College of Life Sciences, Wuhan University, Wuhan 430071, China

${ }^{5}$ Wuhan Recogen Biotechnology Co.,Ltd, Wuhan 430056, China

† These authors contributed equally to this work.

*Corresponding author: Ke Lan (Email: klan@whu.edu.cn)

Yong Hu (Email: yong.hu@rhegen.com)
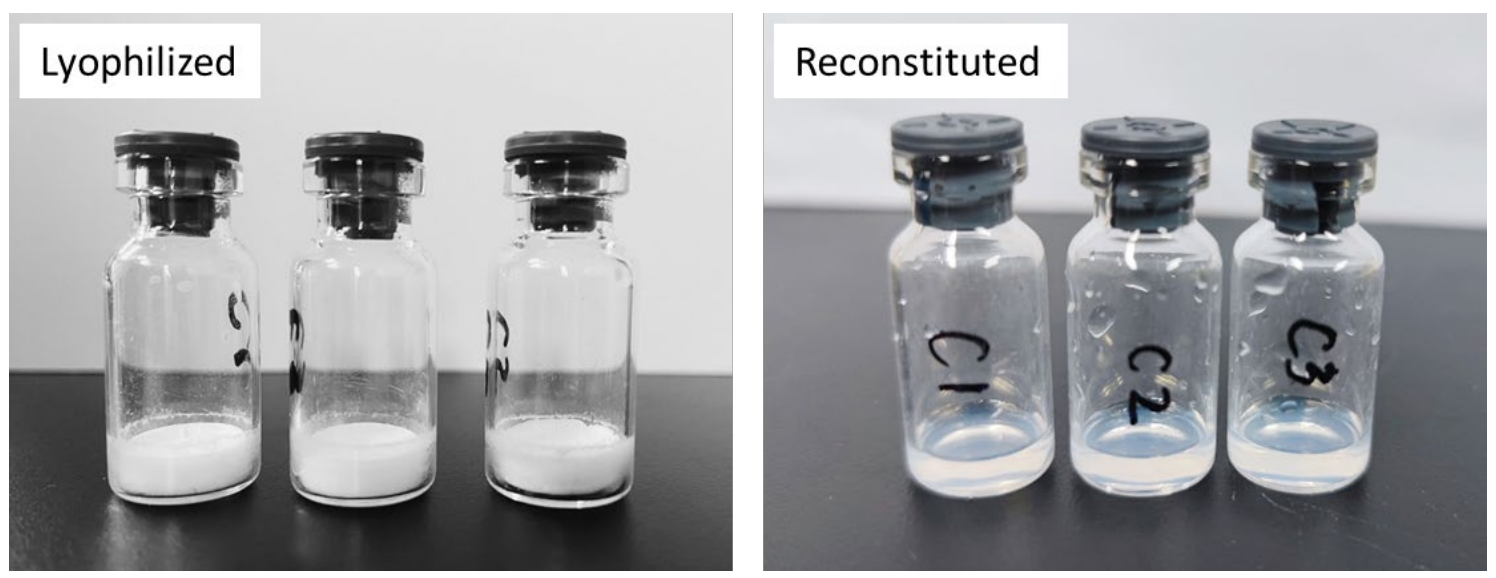

Figure S1. Images of lyophilized mRNA-LNPs and the reconstituted solutions. 

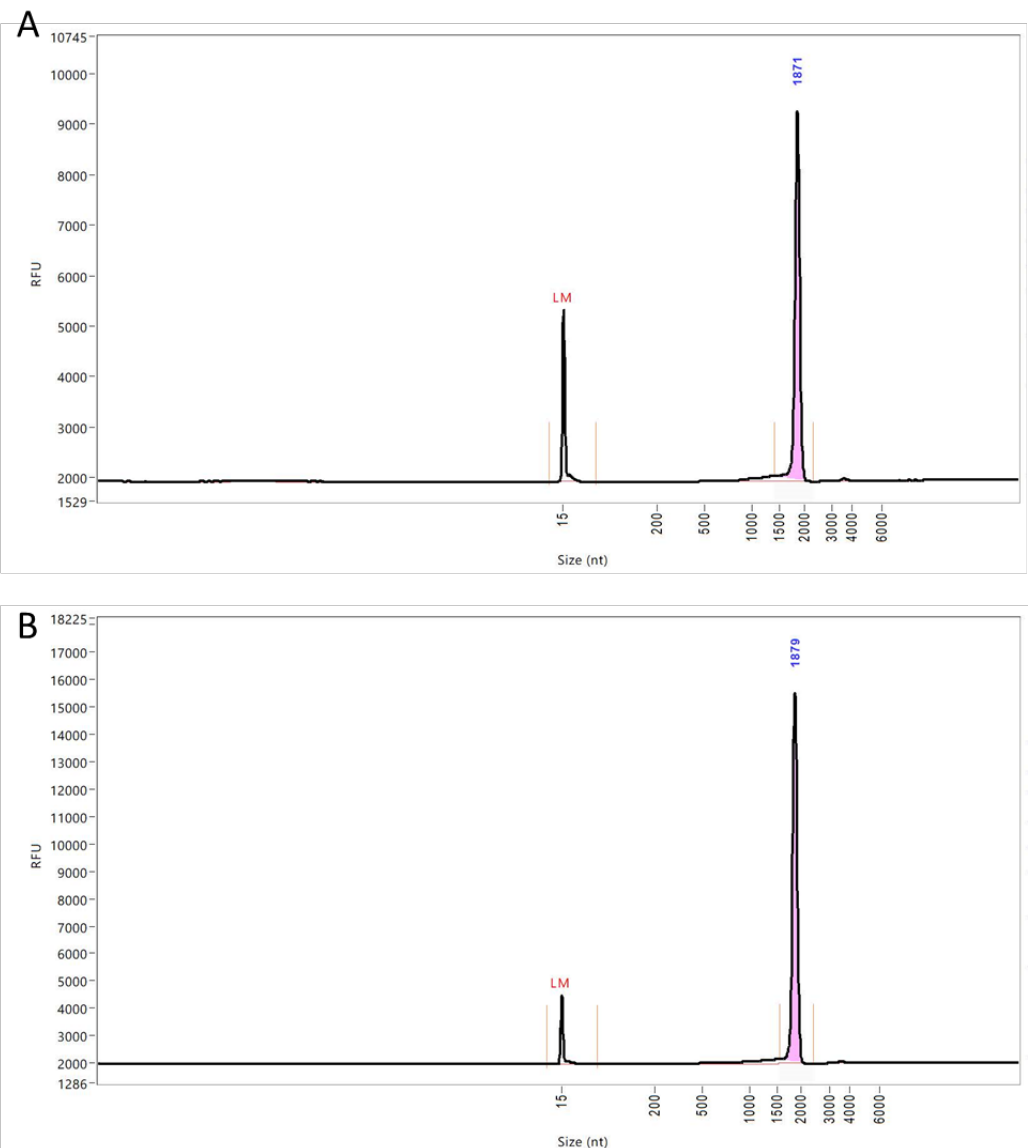

Figure S2. Microfluidic capillary electrophoresis analysis of (A) Omicron mRNA and (B) reconstituted Lyo-mRNA-Omicron. 